САРКОПЕНИЯ ГЛАЗАМИ ЭНДОКРИНОЛОГА

( ) Н.Г. Мокрышева, Ю.А. Крупинова, В.Л. Володичева*, С.С. Мирная, Г.А.Мельниченко

ФГБУ «Национальный медицинский исследовательский центр эндокринологии» Минздрава России, Москва

Одним из синдромов, существенно влияющим на состояние здоровья и жизнь пациента, является саркопения. Саркопения - это мультиэтиологичный синдром со сложным, недостаточно изученным патогенезом, в котором эндокринные факторы играют одну из ведущих ролей. Коморбидность пожилых пациентов создает трудности дифференциальной диагностики имеющейся патологии. Сочетание саркопении с различными эндокринными патологиями, такими как ожирение, остеопороз, гипотиреоз, гипопаратиреоз, дефицит витамина D, приводит к более тяжелому течению заболеваний и худшему прогнозу для качества и продолжительности жизни пациента. При этом вторичный генез саркопении в ряде ситуаций может позволить улучшить состояние пациента путем оценки патогенетических причин и их устранения.

Таким образом, эндокринологу следует уделять более пристальное внимание синдрому саркопении и его коррекции, а также мерам его профилактики на самых ранних стадиях.

КЛЮЧЕВЫЕ СЛОВА: саркопения, витамин D, мышечная сила, мышечная слабость.

\title{
A VIEW AT SARCOPENIA BY ENDOCRINOLOGIST
}

(c) Natalia G. Mokrysheva, Julia A. Krupinova, L. Victoria Volodicheva*, Svetalana S. Mirnaya, Galina A. Melnichenko

Endocrinology Research Centre, Moscow, Russia

Sarcopenia is one of the syndromes that significantly affects the health and life of the patient. Sarcopenia is a polyetiological syndrome with a complex, insufficiently studied pathogenesis, in which endocrine factors play a leading role. Comorbidity of elderly patients creates difficulties in differential diagnosis of existing pathology. The combination of sarcopenia with various endocrine pathologies, such as obesity, osteoporosis, hypothyroidism, hypoparathyroidism, vitamin D deficiency leads to a more severe course of disease and a worse prognosis for the quality and life expectancy of the patient. In this case, in some situations, the patient's condition can be improved by assessing pathogenetic causes and their elimination. Thus, the endocrinologist should pay more attention to the sarcopenia syndrome and its correction, as well as for its prevention at the earliest stages.

KEYWORDS: sarcopenia; vitamin D, muscle strength, muscle weakness.

Одним из синдромов, существенно влияющих на здоровье и жизнь пациента, является саркопения. Саркопения (от греч. sarx - тело, плоть + penia - снижение) состояние, характеризующееся прогрессирующей и генерализованной утратой мышечной массы и силы с повышением риска неблагоприятных событий, таких как ухудшение качества жизни, инвалидизация и смерть (Европейский консенсус по саркопении 2010 года) [1]. Снижение мышечной силы описывал еще Гиппократ, однако термин «саркопения» впервые предложил в 1989 г. американский профессор I. Rosenberg [2]. Диагностические, профилактические и лечебные мероприятия при саркопении должны разрабатываться с учетом повышения коморбидности с возрастом. Это особенно актуально в практике эндокринолога, так как множество эндокринопатий может приводить к формированию данного синдрома.

\section{Эпидемиология}

Распространенность саркопении в популяции по разным источникам варьирует от 4,3\% до 73,3\% и зависит от возрастных критериев включения и особенностей исследуемых групп (пациенты, получающие амбулаторную помощь, и жители домов престарелых имеют значимые отличия). Чаще встречаются данные о распространенности в диапазоне 5-13\% среди лиц 60-70 лет и на уровне $50 \%$ - в группе старше 80 лет [3, 4]. В российской популяции частота составила $13 \%$ у пациентов старше 85 лет [5].

\section{Этиология}

Этиологически саркопению разделяют на первичную и вторичную. Первичную саркопению рассматривают в рамках возрастных изменений мышечной ткани, вторичную - как следствие других заболеваний и патологических состояний (онкологической патологии, сахарного диабета (СД) 2 типа, хронической сердечной недостаточности, гиподинамии и прочего, а также вследствие приема некоторых лекарственных препаратов).

Также обсуждается и генетическая предрасположенность к саркопении. Например, доказана связь генов ACE (кодирует ангиотензинпревращающий фермент), ACTN3 (кодирует альфаактинин 3), MSTN (кодирует миостатин), CNTF (кодирует цилиарный нейротрофический фактор), IGF1 (кодирует ИФР-1), IL-6 (кодирует интерлейкин 6) и VDR (кодирует рецептор витамина D) с силой и/или массой мышечной ткани. Особый интерес для изучения представляют такие гены-кандидаты, как GREM1 (кодирует Gremlin1), TRHR (кодирует рецептор тире- 
отропин-рилизинг гормона), $A C V R 1 B$ (кодирует рецептор активина 1B). В ряде двойных слепых исследований продемонстрировано, что наследуемость некоторых показателей мышечной массы и силы достигает $80 \%[6,7]$.

\section{Патогенез}

Саркопения - это мультиэтиологичный синдром со сложным патогенезом, в котором эндокринные факторы играют одну из ведущих ролей. Патогенез данного состояния изучен недостаточно, однако уже известно, что на развитие саркопении значимое влияние оказывает снижение уровня гормона роста (ГР), инсулиноподобного фактора роста-1 (ИФР-1), половых гормонов, витамина $D$, инсулинорезистентность. В то же время активно изучаются такие факторы, способствующие развитию саркопении, как особенности образа жизни (качество питания, физическая активность, иммобилизация) и пищевой статус. Снижение последнего приводит к недостаточному синтезу белка, развитию дегенеративных процессов, митохондриальной дисфункции и атрофии мышечных волокон, вследствие чего происходит потеря мышечной массы и силы [8]. На развитие саркопении также может влиять течение гестационного периода матери, окружающая среда в раннем детском возрасте, вес тела при рождении и в младенчестве, который прямо связан с мышечной массой и ее функциональными возможностями в старшем возрасте [6].

В основе патогенеза саркопении лежит утрата мышечной массы. Зрелые мышечные волокна состоят из многоядерных клеток, неспособных к делению, поэтому рост и регенерация мышц происходят за счет пролиферации клеток-сателлитов. При саркопении в первую очередь снижается количество клеток-сателлитов и волокон II типа, приводя к невозможности совершения пациентом быстрых движений [9]. Будучи ключевым звеном большинства клеточных процессов (обеспечение энергией, регуляция внутриклеточного гомеостаза кальция, активация клеточной пролиферации), митохондриальная дисфункция и дальнейшая потеря целостности митохондрий в миоцитах приводят к снижению окислительной способности, нарастанию уровня оксидативного стресса, повреждению клеток и развитию атрофии мышечных волокон (рис. 1).

Значительную роль играют системное воспаление, трансформация митохондрий и их взаимное усиление дегенеративного влияния на мышцы. В скелетных мышцах происходит повреждение транспортной цепи электронов, которое может определяться фокально в миоцитах или в дискретных областях отдельной клетки, и приводит к снижению активности цитохрома С-оксидазы и повышению активности сукцинатдегидрогеназы (СДГ) [10].

Доказательства, подтверждающие ключевую роль мутаций митохондриальной ДНК (мтДНК) в патогенезе старения, были получены в эксперименте на животных моделях, у которых имела место ошибка считывания в мтДНК-полимеразе $\gamma$ (PolG), что проявлялось преждевременным старением, характеризующимся алопецией, кифозом, потерей слуха, остеопорозом, саркопенией и сокращением продолжительности жизни. У этих мышей накапливались соматические мутации мтДНК и демонстрировали системную дисфункцию митохондрий,

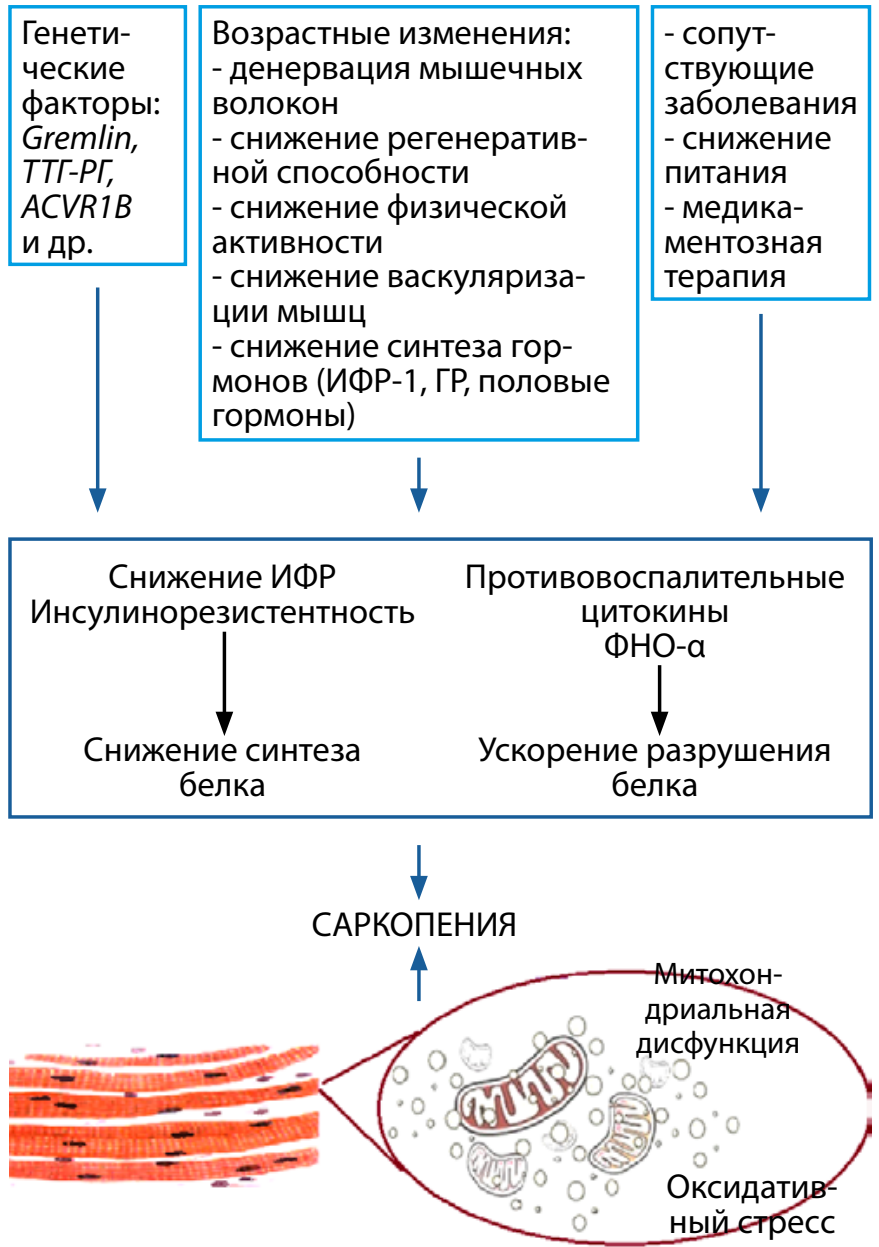

Рис. 1. Этиология и патогенез саркопении.

включая снижение функции дыхательной цепи, ускоренный апоптоз в постмитотических тканях, при этом повышенного уровня окислительного стресса у них не наблюдалось. Интересно, что делеции мтДНК локализуются совместно с мутациями транспортной цепи электронов и при повышении определенного порога значений приводят к нарушению ее активности. В целом эти данные подтверждают гипотезу о том, что делеции мтДНК, накапливаясь в мышечных волокнах, компрометируют митохондриальную биоэнергетику и способствуют развитию атрофии мышц [10].

Другой путь, регулирующий метаболизм митохондрий во время старения, включает ИФР-1. Этот путь осуществляется путем фосфорилирования АТФ-цитрат-лиазы, фермента, катализирующего митохондриальное производное цитрат в оксалоацетат и ацетил-КоА. Предполагается, что возрастное снижение уровня ИФР-1 может нарушать митохондриальную активность посредством снижения АТФ-цитрат-лиазы, способствуя развитию саркопении [10].

Наличие воспалительной реакции также тесно связано с риском развития саркопении. Повышение фактора некроза опухоли-а способствует развитию воспаления через активацию ядерного фактора k-B, что приводит к потере мышечной массы (см. рис. 1) [11].

Таким образом, ИФР-1, механизмы контроля качества митохондрий и воспалительный путь являются основными звеньями, контролирующими мышечную массу.

Повреждения на любом уровне контроля состояния митохондрий сохраняются на протяжении их жизни, та- 
ким образом накапливаются поврежденные органеллы. Как следствие, увеличение отношения АМФ/АТФ и оксидативный стресс активируют катаболический путь, который приводит к атрофии мышц. Этот путь включает в себя как посредников, связанных с аутофагами, так и индукторов мышечного катаболизма (MuRF-1, атрогин), приводящих к деградации митохондрий и белка для обеспечения альтернативных источников энергии [11].

В исследовании, изучавшем взаимосвязь скелетной мышечной массы с сохранностью капиллярной сети при старении, сравнивались данные гистологического исследования мышечной ткани между группами молодых здоровых лиц (средний возраст - 22 года, мужчины $(n=14)$, женщины $(n=5))$ и пожилых лиц (средний возраст - 74 года, мужчины $(\mathrm{n}=22)$, женщины $(\mathrm{n}=6))$. Степень мышечной капилляризации и распределения капилляров определяли методом капиллярных доменов. Окислительную способность мышечных волокон оценивали с помощью количественной гистохимии. Обеспечение капиллярами волокон определялось главным образом размером волокна и лишь в малой степени окислительной способностью. Несмотря на атрофию волокон II типа на $18 \%(p=0,019)$ и снижение количества капилляров на одну клетку на 23\% (p<0,002) у пожилых людей, существенных различий в распределении капилляров между молодыми и пожилыми людьми выявлено не было. При исследовании активности СДГ максимальное потребление кислорода, поддерживаемое капилляром, не сильно различалось между молодыми и пожилыми людьми, но волокна I типа имели, как и ожидалось, более высокую активность СДГ по сравнению с волокнами II типа [12].

\section{КЛИНИЧЕСКАЯ КАРТИНА САРКОПЕНИИ}

Клиническая картина характеризуется снижением объема мышц, их силы и общей активности пациента. Больные отмечают трудности в выполнении привычных действий, выраженную мышечную и общую слабость, боли в мышцах, невозможность самостоятельного передвижения и самообслуживания, что постепенно приводит к инвалидизации и снижению продолжительности жизни. С целью предотвращения таких тяжелых последствий необходимо выявлять данный синдром на самом раннем этапе его развития.

\section{ДИАГНОСТИЧЕСКИЕ МЕТОДЫ}

Заподозрить саркопению возможно с помощью опросников. Наиболее часто используются опросники SarQol (Sarcopenia and Quality of Life - качество жизни при саркопении) и SARC-F (Strength, Assistance with walking, Rise from a chair, Climb stairs and Falls - сила, помощь при ходьбе, подъем со стула, подъем по лестнице и падения). Опросник SarQol разработан в 2015 г. французской научной группой для пациентов старше 65 лет в целях оценки мышечной функции и качества жизни. Он состоит из 22 вопросов, разделенных на модули: физическое и психическое здоровье, активность, состав тела, функциональность, деятельность в повседневной жизни и во время досуга [3]. Опросник SARC-F состоит из 5 вопросов и является скрининговым методом обследова- ния, позволяющим быстро оценить мышечную функцию (чувствительность 4-10\%, специфичность 94-99\%).

Европейская рабочая группа по изучению саркопении у пожилых людей (EWGSOP) рекомендует в качестве стандартного метода оценки физического состояния пациента определять скорость ходьбы на короткое расстояние (4 м). Отрезной точкой является скорость 0,8 м/с, ниже которой необходимо заподозрить саркопению. Вторым этапом следует анализ мышечной силы и массы. Сила мышц оценивается с помощью динамометрии, а масса с помощью денситометрии (двухэнергетической рентгеновской абсорбциометрии, DXA) и биоимпедансного анализа [13]. Для оценки мышечной силы определяют силу сжатия кисти с помощью кистевых динамометров. Наиболее часто используется в зарубежных исследованиях динамометр Jamar. У пациентов с заболеваниями суставов более предпочтителен Martin Vigorimeter, который измеряет давление обхватывающей руки и не зависит от размера кисти. Для более достоверной оценки мышечной силы требуется выполнение двух методик. При проведении проспективного когортного исследования «Хрусталь» в Северо-Западном регионе России был произведен расчет возрастных норм результатов кистевой динамометрии для здоровых людей старше 65 лет. Средняя скорость снижения силы сжатия кисти в российской популяции у мужчин была выше, чем у женщин, и составила примерно 1 кг/год в возрасте от 65 до 76 лет и 0,5 кг/год в возрасте старше 76 лет. Средняя скорость снижения силы сжатия у женщин была 2 кг за 5 лет. Представленные результаты согласуются с результатами других исследований: например, средняя скорость снижения силы сжатия в исследовании Н. Frederiksen и соавт., основанном на анализе трех крупных популяционных исследований, была также выше у мужчин по сравнению

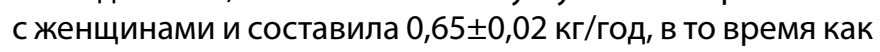

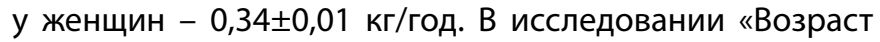
и здоровье женщин Il» (The Women's Health and Aging Study II) скорость снижения силы сжатия у женщин была в среднем 1,10-1,31 кг в возрасте от 70 до 75 и 0,50-0,39 кг в возрасте старше 75 лет [14].

Однако кистевые динамометры не могут достоверно отобразить силу мышц верхних и нижних конечностей. В настоящее время ни один из инструментальных методов не может быть рекомендован для оценки мышечной силы в рутинной клинической практике. Функциональные тесты более информативны, однако также имеют ряд ограничений, например, у пациентов с заболеваниями суставов, неврологическими расстройствами, нарушением координации, головокружением и других. Тест SPPB (Short Physical Performance Battery - характеристика короткой физической активности) позволяет оценить кратковременную физическую активность и включает определение скорости ходьбы на короткое расстояние (4 м), а также 5-кратное вставание со стула и возможность удержания равновесия в положении стоя в течение 10 с [15].

При оценке индекса тощей массы (ИТМ) скелетных мышц с помощью двухэнергетической рентгеновской абсорбциометрии (денситометрии, DXA) рассчитывается частное аппендикулярной мышечной массы (АМM - полученной как сумма тощей массы рук и ног) и роста человека в квадратных метрах, то есть $\mathrm{AMM} / \mathrm{pocT}^{2}$, и измеря- 
Таблица 1. Критерии саркопении по данным биоимпедансного анализа

\begin{tabular}{lcc}
\hline \multicolumn{1}{c}{ Итм } & Муж. & Жен. \\
\hline $\begin{array}{l}\text { Норма } \\
\text { Обратимая }\end{array}$ & $>10,75 \mathrm{kг} / \mathrm{M}^{2}$ & $>6,75 \mathrm{kг} / \mathrm{M}^{2}$ \\
$\begin{array}{l}\text { саркопения } \\
\text { Выраженная } \\
\text { саркопения }\end{array}$ & $8,51-10,75 \mathrm{kг} / \mathrm{M}^{2}$ & $5,76-6,75 \mathrm{kг} / \mathrm{M}^{2}$ \\
\hline
\end{tabular}

ется в кг/м². Саркопения диагностируется при снижении ИТМ более чем на 2 стандартных отклонения, что, по разным источникам, соответствует ИТМ у мужчин менее

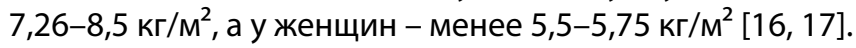

Биоэлектрический импедансный анализ является альтернативой денситометрии, будучи более дешевым, легко воспроизводимым и мобильным методом диагностики. В таблице 1 представлены референсные значения ИТМ для диагностики саркопении.

Вместе с тем биоимпедансный метод исследования недостаточно точен, особенно у пациентов с заболеваниями, проявляющимися отечным синдромом (хроническая сердечная недостаточность, почечная недостаточность и лимфедема). Антропометрические измерения, такие как окружность икроножной мышцы или толщина кожной складки, неточны главным образом потому, что ожирение, отеки, а также изменения в жировой ткани и потеря эластичности кожи, связанные с возрастом, снижают специфичность метода в оценке мышечной массы. Они не рекомендуются для использования в целях диагностики саркопении, но могут быть альтернативными в случае отсутствия других методов [16, 18]. Антропометрический метод недостаточно информативен и при саркопеническом ожирении, включающем критерии саркопении и увеличение объема подкожной жировой клетчатки у мужчин $\geq 25 \%$, у женщин $\geq 35 \%$. При данном состоянии наблюдается жировое перерождение клеток-сателлитов, жировая инфильтрация мышц, которые способствуют снижению силы нижних конечностей и гиподинамии - возникает порочный круг. Однако на данный момент невозможно оценить, что развивается первично: саркопения или ожирение, так как при ожирении наблюдается хроническое воспаление, которое, в свою очередь, приводит к снижению мышечной массы и силы [16].

\section{ОСТЕОСАРКОПЕНИЯ}

Остеосаркопения - это относительно новое понятие, включающее в себя сочетание низкой мышечной массы и сниженной минеральной плотности костной ткани (МПК), связанное со старением и общим патогенезом [19]. Учитывая повышение риска развития как саркопении, так и остеопороза у пожилых лиц, требуется оценка состояния мышечной ткани у таких пациентов [20].

Состав тела пациентов с остеосаркопенией характеризуется снижением мышечной массы, МПК, ИМТ и индекса общего жира по сравнению с пациентами с изолированным остеопорозом или саркопенией. Клинические проявления остеосаркопении характеризуются слабостью, низкой скоростью ходьбы, потерей подвижности. В настоящее время отсутствуют специфические марке- ры остеосаркопении, однако отмечено, что у пациентов с данной патологией уровень тестостерона, витамина D и гемоглобина ниже, чем у пациентов с изолированным остеопорозом или саркопенией.

Помимо возраста, существует ряд других факторов, которые способствуют развитию остеосаркопении. Генетические полиморфизмы GLYAT, METTL21C, MSTN, ACTN3 $P G C-1$ и и MEF-2C связаны с потерей костной и мышечной ткани. Эндокринные заболевания (в основном СД, нарушение функции щитовидной железы и низкий уровень витамина D, половых гормонов, ГР, ИФР-1), алиментарная недостаточность, ожирение и прием кортикостероидов также влияют на развитие остеосаркопении [19]. Отмечено, что саркопения и мышечная слабость, так же как и частота переломов, встречаются чаще у пациентов с СД 2 типа, чем у здоровых людей [21].

Жировая инфильтрация мышц у пожилых вносит свой вклад в развитие остеосаркопении, что, возможно, связано с отрицательным воздействием продукции воспалительных цитокинов костным мозгом и жировой тканью. Установлено, что пациенты с саркопенией и остеопенией имеют высокие концентрации воспалительных цитокинов, преобладание интерлейкина-6 (IL-6) и фактора некроза опухолей альфа (ФНО-а), которые связаны как с системной, так и с локальной липотоксичностью. Кроме того, изменения в костном мозге тесно ассоциированы с изменениями мышечной массы. Скелетная и мышечная ткань взаимодействуют механически, физически, а также биохимически посредством паракринных и эндокринных факторов. В этом процессе существует много возможных путей, которые могут объяснить развитие и прогрессирование остеосаркопении. Один из них включает остеокальцин, который стимулирует пролиферацию $\beta$-клеток, секрецию инсулина и действует непосредственно на скелетную мышцу, коррелируя с мышечной силой. Другим внутренним механизмом, связывающим мышцы и кость, является секреция сосудистого эндотелиального фактора роста (VEGF) с помощью мезенхимальных стромальных клеток костного мозга, что стимулирует пролиферацию миобластов. Мышцы также секретируют несколько гормонов и активных веществ, влияющих на кость, таких как ИФР-1, остеоглицин, ирисин, остеонектин, фактор роста фибробластов-2, IL-6, IL-15 и миостатин. Миостатин является членом надсемейства бета-трансформирующего фактора роста, экспрессируемого в основном в скелетных мышцах. Изучен его эффект на рост мышц и влияние на кость. Дефицит гена MSTN и системное введение ACVR2B-Fc (inhibitor of the Activin Receptor 2B - блокатора рецептора активина 2B) приводят к гипертрофии мышечной ткани и увеличению костной массы [18]. Мышечная ткань также секретирует декорин, который, связывая миостатин, способствует росту мышечной ткани. Обнаружен и его противоопухолевый эффект. При изучении генетически модифицированных животных с выключенным геном DCN, кодирующим декорин, зафиксирован спонтанный рост опухолей. Однако влияние этого белка на физиологию человека изучено недостаточно.

Постменопаузальная остеосаркопения, развитие которой обусловлено генетическими факторами, изменением состава тела, низкой физической активностью, дефицитом эстрогенов и витамина D, приводит 
к снижению мышечной массы, силы, функциональных возможностей, а также к снижению МПК, что в сочетании с коморбидностью пожилых пациентов реализуется в увеличении частоты переломов, инвалидизации и смертности [20].

Также существует такое понятие как остеосаркопеническое ожирение, критериями которого являются снижение МПК по Т-критерию $\leq-1,0$ SD по данным DXA,

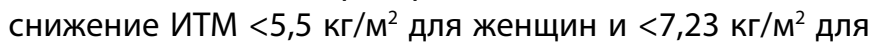
мужчин, повышение массы жира $\geq 38 \%$ для женщин и $\geq 28 \%$ для мужчин, а также соотношение висцерального/подкожного жирового соотношения >1 (для андроидного типа ожирения) или <1 (для гиноидного типа ожирения). Распространенность остеосаркопенического ожирения, по данным перекрестного исследования в Италии, составила 6,86\%, из которых частота гиноидного и андроидного ожирения составила соответственно $2,1 \%$ и $4,7 \%$.

В группе пациентов с остеосаркопеническим висцеральным ожирением (по сравнению с другими когортами) отмечен более высокий уровень С-реактивного белка (>2,34 мг/дл), гликемии (>112 мг/дл), а также более высокий риск переломов (FRAX $>15 \%, r=0,316 ; p<0,05$ ) [22].

\section{САРКОПЕНИЯ И ВИТАМИН D}

Установлено, что витамин D играет важную роль в метаболических процессах в мышечной и костной ткани. Так, снижение его уровня на 10 нг/мл приводит к увеличению частоты развития саркопении в 1,5 раза в течение последующих 5 лет. Однако результаты рандомизированных контролируемых исследований не смогли установить патогенетическую роль витамина D в развитии заболеваний мышечной системы [23].

Было показано, что прием витамина D в дозе от 700 до 800 MЕ/день повышает прочность мышц и костей, снижает риск падений, переломов бедренной кости и смертности, однако оптимальная доза приема витамина D все еще остается неопределенной. Европейское общество по клиническим и экономическим аспектам остеопороза, остеоартрита и скелетно-мышечных заболеваний (ESCEO) также оценивает адекватное потребление витамина D в 800 ME/день для поддержания сывороточного уровня 25-гидроксивитамина D>30 нг/мл (50 нмоль/л) [18]. Баланс между витамином D и паратиреоидным гормоном (ПТГ) является ключевым регулятором мышечной силы: ПТГ способен модулировать работу мышечной ткани через увеличение внутриклеточного кальция.

\section{ЛЕЧЕНИЕ И ПРОФИЛАКТИКА САРКОПЕНИИ}

В настоящее время отсутствует специфическое лечение саркопении, первичной является профилактика. Физические упражнения, особенно постепенно увеличивающиеся силовые нагрузки, являются стимулом для синтеза мышечного белка. Выполнение физических упражнений в течение минимум 20 минут в неделю значительно повышает мышечную и костную массу, силу, снижает функциональные ограничения, предотвращает падения и переломы у пожилых людей. Однако у пациентов с тяжелыми сопутствующими заболеваниями (такими как нейрокогнитивные и сердечные-сосудистые патологии) могут возникнуть сложности в достижении рекомендуемого уровня физической активности [18].

В ходе мета-анализа, включившего 37 исследований, 34 рандомизированных клинических исследования, оценивали влияние физических упражнений на мышечную массу у пожилых людей (почти в 80\% случаев мышечная масса увеличилась с помощью физических упражнений). Влияние дополнительного питания на мышечную массу было зафиксировано только в 23,5\% случаев. Мышечная сила увеличилась в 82,8\% исследований после повышения физической активности, а пищевые добавки показали дополнительные преимущества только в небольшом количестве исследований (22,8\%). Таким образом, физические упражнения оказывают положительное влияние на мышечную массу и силу или физическую работоспособность у здоровых лиц в возрасте от 60 лет и старше [24].

Белок пищи оказывает косвенное влияние на ключевые регуляторные белки и факторы роста, связанные со здоровьем мышц и костей, за счет увеличения абсорбции кальция, подавления ПТГ и увеличения секреции ИФР-І (костного и мышечного анаболического стимулятора). Потребление белка более 0,8 мг/кг массы тела в день, в основном животного происхождения, усиливает анаболизм мышечного белка наряду с защитой от возрастной потери мышц и костной ткани. У пациентов в возрасте 70-79 лет содержание белка в пище в количестве 1,1 г/кг массы тела в день приводит к отсутствию мышечной потери при 3-летнем наблюдении.

ESCEO рекомендует потребление белка с пищей от 1,0 до 1,2 г/кг массы тела в день, минимум 20-25 г чистого белка в каждой порции. Европейское общество клинического питания и обмена веществ (ESPEN) и Исследовательская группа PROT-AGE рекомендуют такое же потребление белка для здоровых людей старшего возраста, от 1,2 до 1,5 г/кг массы тела в день, а для пациентов с тяжелыми заболеваниями и недостаточным питанием - свыше 2 г/кг в сутки [18]. Пациенты с хронической болезнью почек (ХБП) додиализных стадий для профилактики саркопении должны снизить потребление белка до 0,2-0,5 г/кг в сутки (при СКФ <20 мл/мин/1,73 м²) вследствие возможного развития метаболического ацидоза [25]. Кроме того, потребление белка совместно с физическими упражнениями улучшает синтез мышечного белка и положительно влияет на состав тела, способствуя снижению жировой массы, увеличению или поддержанию мышечной ткани и сохранению кости [18] (табл. 2).

Таким образом для профилактики развития саркопении следует придерживаться рекомендаций ESCEO об оптимальном потреблении белка с пищей 1,0-1,2 г/кг тела в день с содержанием по меньшей мере 20-25 г белка на каждый основной прием пищи, об адекватном потреблении витамина D 800 ME/сут, а также потреблении кальция в дозе 1000 мг/сут в сочетании с регулярными физическими упражнениями 3-5 раз в неделю [26].

Активно изучаются возможности патогенетического лечения саркопении. Рандомизированное клиническое исследование 2 фазы показало, что введение антител к миостатину приводит к увеличению аппендикулярной мышечной массы по сравнению с плацебо. Однако остаются 
Таблица 2. Рекомендуемое количество потребления белка в сутки различным группам людей

\section{Критерий}

Количество потребляемого белка

в сутки, г/кг массы тела

\section{Здоровые люди старшего возраста}

Пациенты с острыми и хроническими заболеваниями

Саркопения первичная (пожилые пациенты с недостаточным питанием)

Пациенты с ХБП С5 (до начала гемодиализа)

ХБП С1-3 А2

ХБП С1-3 АЗ

ХБП С4

ХБП С5

ХБП С5Д (гемодиализ)

ХБП С5Д (перитонеальный диализ)

ХБП (после трансплантации почки)
$1,0-1,2$

$1,2-1,5$

свыше 2

$$
0,2-0,5
$$

не более 1,0

$0,6-0,8$

$0,6-0,8$

$0,2-0,5$

не более 1,2

не более 1,4

$0,8-1,0$ нерешенными вопросы о клинической эффективности и безопасности этого метода лечения, поскольку миостатин также наблюдается в сердечной мышечной ткани и его ингибирование потенциально может привести к развитию кардиомиопатии. Исследование антител к миостатину все еще находится в экспериментальной фазе испытаний для оценки эффективности и безопасности данного лечения.

В экспериментальных условиях показано, что ACVR2B/ Fc, блокатор рецептора активина 2B, сохраняет мышечную массу и увеличивает костную в животных моделях с несовершенным остеогенезом и мышечной дистрофией [27]. Применение цитруллина (непротеиногенная аминокислота), как показали исследования на крысах и молодых взрослых людях, увеличивает мышечную массу, синтез белка, а также липолиз в адипоцитах [28].

В результате экспериментального введения ГР мышам после длительной иммобилизации конечности наблюдалось увеличение мышечной массы, а введение ПтГ привело к улучшению микроархитектоники костей, повышению МПК. Комбинированная терапия ГР и ПТГ оказала более выраженное влияние на параметры костной ткани, не улучшив при этом показатели мышечной массы по сравнению с монотерапией ГР. Однако клинические исследования, проведенные на людях, не дали ожидаемого результата и по применению ГР: мышечная масса увеличилась незначительно [29, 30].

Различные другие фармакологические препараты, такие как тестостерон, ИФР-1, витамин D, ПТГ и т.д., оцениваются как потенциальные средства для лечения саркопении, однако необходимый эффект не был достигнут [18]. Таким образом, в настоящее время можно говорить только о профилактике данного состояния, в которую входят адекватные физические нагрузки и питание с достаточным потреблением белка.

\section{ЗАКЛЮЧЕНИЕ}

Наиболее изученным последствием саркопении является повышение смертности: метаанализ 17 исследований показал, что риск смерти в 4 раза выше у пациентов с саркопенией, риск снижения функциональных возможностей мышц или инвалидности в 3 раза выше, чем у лиц того же возраста без данного синдрома [3]. Это подчеркивает клиническую и социальную значимость саркопении, необходимость тщательного изучения данного состояния, поиск ее предикторов и эффективного лечения.

\section{ДОПОЛНИТЕЛЬНАЯ ИНФОРМАЦИЯ}

Конфликт интересов. Авторы декларируют отсутствие явных и потенциальных конфликтов интересов, связанных с публикацией настоящей статьи.

Участие авторов. Все авторы внесли значимый вклад в проведение поисково-аналитической работы и подготовку статьи, прочли и одобрили финальную версию перед публикацией.

\section{СПИСОК ЛИТЕРАТУРЫ | REFERENCES}

1. Cruz-Jentoft AJ, Baeyens JP, Bauer JM, et al. Sarcopenia: European consensus on definition and diagnosis: Report of the European Working Group on Sarcopenia in Older People. Age Ageing 2010;39(4):412-423. doi: 10.1093/ageing/afq034.

2. Rosenberg I.H. Epidemiologic and methodologic problems in determining nutritional status of older persons. Proceedings of a conference. Albuquerque, New Mexico, October 19-21, 1988. Am. J. Clin. Nutr. 1989;50(5 Suppl):1121-1235.

3. Wright JM, Beaudart C, Zaaria M, et al. Health Outcomes of Sarcopenia: A Systematic Review and Meta-Analysis. PLoS One. 2017;12(1):e0169548. doi: 10.1371/journal.pone.0169548.

4. Morley JE, Anker SD, von Haehling S. Prevalence, incidence, and clinical impact of sarcopenia: facts, numbers, and epidemiology-update
2014. Journal of Cachexia, Sarcopenia and Muscle. 2014;5(4):253-259. doi: 10.1007/s13539-014-0161-y.

5. Safonova Y, Zotkin E, Lila A. Prevalence of sarcopenia in elderly northwestern region of the Russian Federation. WCO-IOF-ESCEO. World Congress on Osteoporosis, Osteoarthritis and Musculoskeletal Diseases; 2018 April 19-22, Krakow, Poland. Springer; 2018. p. 238. doi: 10.13140/RG.2.2.22677.01768

6. Scott D. Reducing the burden of sarcopenia: a healthy lifestyle throughout the lifetime D. WCO-IOF-ESCEO. World Congress on Osteoporosis, Osteoarthritis and Musculoskeletal Diseases; 2018 April 19-22, Krakow, Poland. Springer; 2018. p. 99.

7. Tan L-J, Liu S-L, Lei S-F, et al. Molecular genetic studies of gene identification for sarcopenia. Hum. Genet. 2011;131(1):1-31. doi: 10.1007/s00439-011-1040-7. 
8. Ali S, Garcia JM. Sarcopenia, Cachexia and Aging: Diagnosis, Mechanisms and Therapeutic Options - A Mini-Review. Gerontology. 2014:60(4):294-305. doi: 10.1159/000356760.

9. Sayer AA, Robinson SM, Patel HP, et al. New horizons in the pathogenesis, diagnosis and management of sarcopenia. Age Ageing. 2013:42(2):145-150. doi: 10.1093/ageing/afs191.

10. Picca A, Calvani R, Bossola M, et al. Update on mitochondria and muscle aging: all wrong roads lead to sarcopenia. Biol. Chem. 2018;399(5):421-436. doi: 10.1515/hsz-2017-0331.

11. Radic M. Autoimmune sarcopenia - current knowledge and perspective. WCO-IOF-ESCEO. World Congress on Osteoporosis, Osteoarthritis and Musculoskeletal Diseases; 2018 April 19-22, Krakow, Poland. Springer; 2018. p. 135

12. Barnouin Y, McPhee JS, Butler-Browne G, et al. Coupling between skeletal muscle fiber size and capillarization is maintained during healthy aging. Journal of Cachexia, Sarcopenia and Muscle. 2017;8(4):647-659. doi: 10.1002/jcsm.12194.

13. Ji H-M, Han J, Won Y-Y. Sarcopenia and Osteoporosis. Hip \& Pelvis. 2015;27(2):72. doi: 10.5371/hp.2015.27.2.72

14. Турушева А.В., Фролова Е.В., Дегриз Я.-М. Расчет возрастных норм результатов кистевой динамометрии для здоровых людей старше 65 лет в Северо-Западном регионе России: результаты проспективного когортного исследования «Хрусталь» // Российский семейный врач. - 2017. - Т. 21. - №4. - С. $29-35$. [Turusheva AV, Frolova EV, Degryse J-M. Development of reference ranges of handgrip strength among healthy adults $65+$ in Northwest Russia: a prospective population-based cohort Crystal study. Russian Family Doctor. 2017;21(4):29-35. (In Russ.)] doi: 10.17816/ rfd2017429-35.

15. Bauer J. M. Principles for the standardization of the assessment of muscle strength and power. WCO-IOF-ESCEO. World Congress on Osteoporosis, Osteoarthritis and Musculoskeletal Diseases; 2018 April 19-22, Krakow, Poland. Springer; 2018. p. 75.

16. Rolland Y, Czerwinski S, van Kan GA, et al. Sarcopenia: Its assessment, etiology, pathogenesis, consequences and future perspectives. The Journal of Nutrition Health and Aging. 2008;12(7):433-450. doi: 10.1007/bf02982704.

17. Carnevale V, Castriotta V, Piscitelli PA, et al. Assessment of Skeletal Muscle Mass in Older People: Comparison Between 2 Anthropometry-Based Methods and Dual-Energy X-ray Absorptiometry. J. Am. Med. Dir. Assoc. 2018;19(9):793-796. doi: 10.1016/j.jamda.2018.05.016.
18. Hirschfeld HP, Kinsella R, Duque G. Osteosarcopenia: where bone, muscle, and fat collide. Osteoporos. Int. 2017;28(10):2781-2790. doi: 10.1007/s00198-017-4151-8.

19. Frisoli A. Clinical and biochemical phenotype of osteosarcopenia. WCO-IOF-ESCEO World Congress on Osteoporosis, Osteoarthritis and Musculoskeletal Diseases; 2017 March 23-26, Florence, Italy. Springer; 2017. p.106

20. Sirola J, Kröger H. Similarities in Acquired Factors Related to Postmenopausal Osteoporosis and Sarcopenia. Journal of Osteoporosis. 2011:2011:1-14. doi: 10.4061/2011/536735.

21. Trierweiler H, Kisielewicz G, Hoffmann Jonasson T, et al. Sarcopenia: a chronic complication of type 2 diabetes mellitus. Diabetol. Metab. Syndr. 2018;10(1). doi: 10.1186/s13098-018-0326-5.

22. Perna S, Spadaccini D, Nichetti M, et al. Osteosarcopenic Visceral Obesity and Osteosarcopenic Subcutaneous Obesity, Two New Phenotypes of Sarcopenia: Prevalence, Metabolic Profile, and Risk Factors. Journal of Aging Research. 2018;2018:1-8. doi: 10.1155/2018/6147426.

23. Bruyère O. Vitamin D and muscle function. WCO-IOF-ESCEO. World Congress on Osteoporosis, Osteoarthritis and Musculoskeletal Diseases; 2018 April 19-22, Krakow, Poland. Springer; 2018. p.124.

24. Beaudart C. Outcomes of the IOF-ESCEO sarcopenia working groups. WCO-IOF-ESCEO. World Congress on Osteoporosis, Osteoarthritis and Musculoskeletal Diseases; 2018 April 19-22, Krakow, Poland. Springer; 2018. p.77-78.

25. National Kidney F. K/DOQI clinical practice guidelines for bone metabolism and disease in chronic kidney disease. Am. J. Kidney Dis. 2003:42(4 Suppl 3):S1-201.

26. Reginster J.-Y. Dairy products in the prevention and treatment of sarcopenia: outcomes of the ESCEO Working Groups. WCO-IOF-ESCEO. World Congress on Osteoporosis, Osteoarthritis and Musculoskeletal Diseases; 2018 April 19-22, Krakow, Poland. Springer; 2018. p. 78.

27. Barreto R, Kitase $Y$, Matsumoto T, et al. ACVR2B/Fc counteracts chemotherapy-induced loss of muscle and bone mass. Sci. Rep. 2017;7(1). doi: 10.1038/s41598-017-15040-1.

28. Aubertin-Leheudre M. Citruline and muscle function. WCO-IOF-ESCEO World Congress on Osteoporosis, Osteoarthritis and Musculoskeletal Diseases; 2018 April 19-22, Krakow, Poland. Springer; 2018. p.124.

29. Brent MB, Brüel A, Thomsen JS. PTH (1-34) and growth hormone in prevention of disuse osteopenia and sarcopenia in rats. Bone. 2018;110:244-253. doi: 10.1016/j.bone.2018.02.017.

\section{ИНФОРМАЦИЯ ОБ АВТОРАХ [AUTHORS INFO]}

*Володичева Виктория Леонидовна, клинический ординатор [Victoriya L. Volodicheva, MD]; адрес: Россия, 117036, Москва, ул. Дм. Ульянова, д. 11 [address: 11 Dm. Ulyanova street, 117036 Moscow, Russia]; ORCID: https://orcid.org/0000-0003-3355-2744; eLibrary SPIN: 7175-0736; e-mail: volodicheva.v.l@gmail.com

Мокрышева Наталья Георгиевна, д.м.н., исполнительный директор [Natalia G. Mokrysheva, MD, PhD]; ORCID: http://orcid.org/0000-0002-9717-9742; eLibrary SPIN: 5624-3875; e-mail: nm70@mail.ru

Крупинова Юлия Александровна [Julia A. Krupinova, MD]; ORCID: http://orcid.org/0000-0001-7963-5022; eLibrary SPIN: 6279-8247; e-mail: j.krupinova@gmail.com

Мирная Светлана Сергеевна, к.м.н. [Svetlana S. Mirnaya, MD, PhD]; ORCID: http://orcid.org/0000-0002-1341-0397; eLibrary SPIN: 1968-7706; e-mail: svetlanamirnaya@yahoo.com

Мельниченко Галина Афанасьевна, д.м.н., профессор, академик PAH [Galina A. Melnichenko, MD, PhD, Professor]; ORCID iD: 0000-0002-5634-7877; eLibrary SPIN: 8615-0038; e-mail: teofrast2000@mail.ru

\section{ЦИТИРОВАТЬ:}

Мокрышева Н.Г., Крупинова Ю.А., Володичева В.Л., Мирная С.С., Мельниченко Г.А. Саркопения глазами эндокринолога // Ожирение и метаболизм. - 2018. - Т.15. - №. 3 - С. 21-27. doi: 10.14341/OMET9792

\section{TO CITE THIS ARTICLE:}

Mokrysheva NG, Krupinova YA, Volodicheva VL, Mirnaya SS, Melnichenko GA. A view at sarcopenia by endocrinologist. Obesity and metabolism. 2018;15(3):21-27. doi: 10.14341/OMET9792 